but there is a disconnect between this and the support provided in the hospice in-patient setting.

Aim To assess whether rehabilitative palliative care (RPC) enables HPPC to be integrated in the in-patient setting.

Method Using participatory action research, a co-operative inquiry group planned how to integrate RPC in a UK Hospice in-patient unit paying attention to the facilitators and barriers.

The findings from this study and the literature were examined to identify the alignment and dissonance between HPPC and RPC.

Results A post intervention review indicated that RPC had been implemented, but there was conflict, also identified in the literature, between a model perceived to be focused on caring (palliative), and one based on enabling (rehabilitation).

Factors demonstrating the similarities between HPPC and RPC were presented:

- democracy, empowerment and participation were underpinning principles

- focused on enablement, control, choice and independence where self-esteem, self-determination and self-reliance coexisted with high levels of physical dependence

- participatory models with an emphasis on social interaction

- encouraged health care professionals to relinquish an expertled approach to enable patients to become active participants in their care

- interdisciplinary - involving all health and social care professionals, patients, families, communities and volunteers.

Claims challenging whether RPC could be considered as HPPC, e.g. lack of community involvement, a failure to address death education, RPC was death denying and perpetuated a clinical model of death and dying were discussed and alternative perspectives presented.

Conclusion Despite its close associations with community initiatives, the principles of HPPC can be integrated in a hospice in-patient setting using RPC.

\section{DOCUMENTATION OF RESUSCITATION DISCUSSIONS IN 2013 AND 2015: PRE AND POST TRACEY JUDGEMENT - A NATIONAL AUDIT OF PRACTICE}

Hazel Coop, Charlotte Chamberlain, Nikhil Sanyal, Alistair Duncan, Felicity Dewhurst, on behalf of UK PRC. UK Palliative Trainee Research Collaborative (UK_PRC)

10.1136/spcare-2020-PCC.73

Background In 2014 the Tracey judgement resulted in amended Resuscitation Council guidance, recommending that patients and relatives should be involved in resuscitation discussions unless it would cause harm. This project aimed to examine whether improved hospice resuscitation discussion documentation between 2013 and 2015 found regionally by the West Midlands palliative trainee collaborative (WM CARES) was evident in hospice and hospital settings across the United Kingdom.

Methods National resuscitation standards in 2013 and 2015 from the UK Resuscitation Council were identified. Data collectors were recruited by the UK Palliative trainee Research Collaborative (UK PRC). Do not attempt resuscitation decision (DNACPR) documentation was sought for 30 deaths in 2013 and 30 deaths in 2015 from each participating hospice and hospital. An excel database was used for data collection and analysis.

Results Data for 805 patients across 17 sites (5 hospitals and 12 hospices) in England and Wales were collected. 2013 data were missing from one site and 130 patients were excluded as did not meet audit criteria. 40\% (157) of patients had documented resuscitation discussions in 2013 compared to $60 \%$ (271) in 2015. More documented DNACPR discussions occurred with patients in hospices $(57 \%, 330)$ compared with patients in hospital $(42 \%, 98)$. However, relatives in hospital settings $(73 \%, 168)$ were more likely to be documented as involved in discussions compared with relatives in hospice settings $(58 \%, 334)$. The most common reasons cited for not discussing DNACPR with patients (2013 and 2015) were: lack of capacity or advanced illness. 20\% (171) did not document a reason for no resuscitation discussion, but this was 75\% less (120 to 30) in 2015 compared with 2013.

Conclusions DNACPR discussions appear to be happening more frequently in 2015, compared with 2013, but there is clearly room for improvement in hospices and hospitals.

\section{A CLINICAL AUDIT TO EVALUATE HOW DNACPR DECISIONS ARE SHARED ON DISCHARGE FROM TY OLWEN - A SPECIALIST PALLIATIVE CARE UNIT IN WALES}

Alice Crabtree, Jessica Gutjahr, Edward Presswood. NHS

\subsection{6/spcare-2020-PCC.74}

Background The 'Sharing and Involving' Clinical Policy for Do Not Attempt Cardio Pulmonary Resuscitation (DNACPR) for Adults in Wales sets standards for how DNACPR decisions are documented and shared. The aim was to find out if $\mathrm{Ty}$ Olwen, a 14 bed Inpatient Palliative Care Unit in South Wales, meets the specified standards.

Method In October 2016, a new DNACPR discharge checklist was created to improve practice and enable collection of prospective data for this audit. Data collection took place in December 2018, of all discharged between October 2016 and November 2018. Following an education intervention, the audit data collection was repeated in August 2019 for discharges between January 2019 and July 2019. Compliance with each standard was calculated as a percentage.

Results Between October 2016 and November 2018, 64 patients were discharged from Ty Olwen. There were $58 \mathrm{com}$ pleted checklists during this period. 93\% of DNACPR forms were signed or countersigned by a senior responsible clinician. $90 \%$ of patients were given a copy of the DNACPR form prior to discharge. $86 \%$ of DNACPR forms were forwarded to the patient's GP. The audit data and our recommendations were presented at the Ty Olwen clinical governance meeting. Re-audit in August 2019 showed limited improvement to these figures.

Conclusion Ty Olwen is not fully compliant with the policy standards. Other hospices and inpatient units in Wales, and across the UK, may face similar challenges. This audit, and the resulting recommendations, should be considered by palliative care teams throughout Wales to measure and improve compliance with the national policy. 